\title{
BMJ Open Web-based intervention to reduce psychological barriers to insulin therapy among adults with non-insulin-treated type 2 diabetes: study protocol for a two- armed randomised controlled trial of 'Is insulin right for me?'
}

\author{
Elizabeth Holmes-Truscott (D) , ${ }^{1,2}$ Edith E Holloway (D) , ${ }^{1,2}$ Hanafi M Husin (1) ,1,2 \\ John Furler (D) , ${ }^{3}$ Virginia Hagger (D) , ${ }^{4}$ Timothy C Skinner (D) ,, Jane Speight (D) ${ }^{1,2}$
}

To cite: Holmes-Truscott E, Holloway EE, Husin HM, et al. Web-based intervention to reduce psychological barriers to insulin therapy among adults with non-insulin-treated type 2 diabetes: study protocol for a two-armed randomised controlled trial of 'Is insulin right for me?. BMJ Open 2022;12:e051524. doi:10.1136/ bmjopen-2021-051524

- Prepublication history and additional supplemental material for this paper are available online. To view these files, please visit the journal online (http://dx.doi.org/10.1136/ bmjopen-2021-051524).

Received 22 March 2021 Accepted 02 February 2022

Check for updates

(c) Author(s) (or their employer(s)) 2022. Re-use permitted under CC BY-NC. No commercial re-use. See rights and permissions. Published by BMJ.

For numbered affiliations see end of article.

Correspondence to Elizabeth Holmes-Truscott; etruscott@acbrd.org.au

\section{ABSTRACT}

Introduction Psychological barriers to insulin therapy are associated with the delay of clinically indicated treatment intensification for people with type 2 diabetes (T2D), yet few evidence-based interventions exist to address these barriers. We describe the protocol for a randomised controlled trial (RCT) examining the efficacy of a novel, theoretically grounded, psychoeducational, web-based resource designed to reduce psychological barriers to insulin among adults with non-insulin treated T2D: 'Is insulin right for me?'.

Methods and analysis Double-blind, parallel group RCT. A target sample of $\mathrm{N}=392$ participants $(\mathrm{n}=196 / \mathrm{arm})$ will be randomised (1:1) to 'Is insulin right for me?' (intervention) or widely available online resources (control). Eligible participants include adults (18-75 years), residing in Australia, currently taking oral hypoglycaemic agents to manage T2D. They will be primarily recruited via invitations and reminders from the national diabetes registry (from a purposefully selected sample of $\mathrm{N} \geq 12000$ ). Exclusion criteria: experience of selfadministered injectable; previously enrolled in pilot RCT; 'very willing' to start insulin as baseline. Outcomes will be assessed via online survey at 2 weeks and 6 months. Primary outcome between-group: difference in mean negative Insulin Treatment Appraisal Scores (ITAS negative) at 2-week and 6-month follow-up. Secondary outcomes: between-group differences in mean positive insulin appraisals (ITAS positive) and percentage difference in intention to commence insulin at follow-up time points. All data analyses will be conducted according to the intention-to-treat principle.

Ethics and dissemination Deakin University Human Research Ethics Committee (2020-073). Dissemination via peer-reviewed journals, conferences and a plain-language summary.

Trial registration number ACTRN12621000191897; Australian and New Zealand Clinical Trials Registry.

\section{INTRODUCTION}

Type 2 diabetes (T2D) is a progressive condition that requires timely adjustment of treatment to achieve and maintain optimal glucose outcomes $^{1-3}$ and prevent or delay the onset of

\section{Strengths and limitations of this study}

- 'Is insulin right for me?' is the first self-directed, theoretically grounded web-based intervention targeting salient psychological barriers to insulin.

- This fully powered randomised controlled trial will provide evidence of the impact of 'Is insulin right for me?' to reduce negative insulin appraisals and increase intention to initiate insulin among adults with non-insulin-treated type 2 diabetes recruited via a national diabetes registry.

- Comprehensive data collection, including demographic and clinical characteristics, psychosocial outcomes and website analytics, will enable process evaluation analyses.

- Limitations include the self-selected sample, which may lead to an under-representation of those hardest to reach or most at need (ie, those not at all willing to commence insulin).

- Furthermore, this study is not designed to identify the intervention's impact on actual timely insulin uptake nor feasibility of implementation within clinical care.

micro and macrovascular complications. ${ }^{45} \mathrm{~A}$ staged approach to pharmacological management of glucose in T2D is recommended, ${ }^{1-3}$ including early consideration and initiation of insulin where glycaemic outcomes are above target (typically haemoglobin A1c (HbA1c) $>7 \%, 53 \mathrm{mmol} / \mathrm{mol}^{2}$ ) despite maximal dose of non-insulin medicines. However, vast literature suggests that treatment adjustment, including insulin initiation, is often delayed well beyond the point of clinical need. ${ }^{67}$ For example, a large-scale $(\mathrm{N} \geq 80000)$, retrospective study conducted in the UK, identified $\mathrm{HbA1c}$ at insulin initiation for people with $\mathrm{T} 2 \mathrm{D}$ was $\geq 8.7 \%(72 \mathrm{mmol} / \mathrm{mol})$ with a 
median time until insulin initiation of $\geq 6$ years. ${ }^{8}$ Finally, a recent Australian primary care-based prospective study identified that, among adults with T2D for whom insulin was clinically indicated (HbAlc $\geq 7.5 \% / 58 \mathrm{mmol} / \mathrm{mol}$, with maximal oral therapy), receiving usual care, only $31 \%$ had initiated insulin within 24 months. ${ }^{9} 10$

Reasons for the delay of treatment intensification are multifaceted, ${ }^{71112}$ and effective interventions targeting barriers to insulin use are required. ${ }^{13-15}$ At a systemic or health professional level, promising results have been shown using multidisciplinary models of care (eg, an enhanced practice nurse role within primary care setting $^{9}$ ), effective consultation strategies (eg, collaborative approach to care ${ }^{16}$ ) and insulin-specific structured education programmes. ${ }^{17}{ }^{18}$ However, there is a parallel need for interventions, which directly target the psychological barriers (negative beliefs and attitudes) to insulin held by the person with T2D. Our prior research demonstrated, independent of an optimised model of primary care ('stepping up'), attitudes towards insulin were associated with hypothetical willingness to initiate insulin, which, in turn, predicted actual insulin use 12 months later. ${ }^{14}{ }^{19}$ Elsewhere, qualitative research with people with T2D attending an insulin-specific education programme identified an unmet need for psychological barriers to insulin to be addressed appropriately. ${ }^{20}$ Furthermore, unaddressed negative insulin appraisals may have longlasting impact on the optimal use of insulin and/or emotional well-being following insulin initiation. ${ }^{21-23}$ Such psychological barriers to insulin use include, for example, worries about performing injections, potential pain and side effects as well as feelings of guilt and selfblame about the onset of the condition and/or the need for treatment progression. ${ }^{24}$

Few evidence-based interventions targeting psychological barriers to insulin have been developed and fewer still are evaluated adequately, or implemented beyond research studies. ${ }^{172526}$ Furthermore, preliminary data from relevant clinic-based and insulin starts group-education interventions suggest low intervention uptake among people with T2D. ${ }^{1726}$ In addition to common barriers to outpatient clinic and structured education programme attendance discussed elsewhere, ${ }^{27} 28$ this low uptake may be in part due to individuals concern that participation would lead to insulin acceptance.$^{26}$ Furthermore, health professionals report limited time and resources to facilitate insulin starts, ${ }^{12}$ and express concerns about the added burden of intervention delivery on their already limited time. ${ }^{26}$ Effective interventions that complement clinical care (but are not reliant on a health professional for delivery) have the potential to be acceptable to both people with T2D and their health professionals.

Given the sheer size of the population with T2D, the potential for scalable implementation is also an important consideration. The internet may be an ideal platform to reach those with T2D with concerns about insulin, as it also allows for anonymity in information seeking. One-third of Australian adults with T2D and suboptimal HbA1c report seeking online health information in a past 12 periods. ${ }^{29}$ Furthermore, online interventions for the management of T2D with clear theoretical groundings and based on behaviour change techniques (BCTs) show favourable outcomes. ${ }^{30}$ While peak health bodies publish resources online about T2D treatments, these materials are not typically theoretically informed, do not use evidence-based BCTs $^{3132}$ and are rarely developed in consultation with, or evaluated among, people with T2D. Furthermore, these resources are rarely targeted at addressing salient psychological barriers to treatment use.

In line with UK Medical Research Council guidance for developing and evaluating complex intervention, we developed a theoretically grounded, psychoeducational, web-based resource for people with non-insulin-treated T2D designed to reduce salient psychological barriers to insulin therapy: 'Is insulin right for me?'. ${ }^{33}$ A pilot study demonstrated feasibility of a two-arm randomised controlled trial (RCT) design to test intervention efficacy, compared with widely available online informational resources as well as acceptability of the intervention among adults with T2D. ${ }^{34}$

This protocol describes the design of a double-blinded, parallel group, individually RCT (two-arms, 1:1 ratio), comparing 'Is insulin right for me?' (intervention) with widely available online text-based resources about insulin (control) among adults with non-insulin-treated T2D. We hypothesise an immediate (2 weeks) and sustained (6 months) positive effect of the intervention, compared with control, on negative insulin appraisals. We also expect the intervention to be acceptable to users and to be associated with immediate and sustained improvement in positive insulin appraisals and hypothetical willingness to begin insulin therapy.

\section{METHODS AND ANALYSIS \\ Study setting}

Participation in this Australian study, including provision of informed content, data collection and intervention exposure, is completely online, using personal computers/mobile devices.

\section{Participants and recruitment}

Potential participants will be enrolled in the study only if they meet all the inclusion criteria and none of the exclusion criteria. Inclusion criteria: aged 18 to 75 years; diagnosed with T2D; use of oral hypoglycaemic agents; able to read/write in English and capable of providing informed consent; residing in Australia; access to an internet-enabled computer or tablet device for the duration of the study. Exclusion criteria: diagnoses of diabetes other than T2D; current or prior experience of self-administered injectable treatment for any illness or condition (including diabetes); unable to read/write in English; unable to use/access internet-enabled devices; enrolled as a participant in the pilot $\mathrm{RCT}^{34}$; reports being 'very willing' to initiate insulin therapy (measured using 
a single-item 'hypothetical willingness' questionnaire ${ }^{35}$ ), that is, rendering it impossible to record improvement in this outcome measure.

The primary method of recruitment will be via invitation from the National Diabetes Services Scheme (NDSS). A random sample of $\geq 12000$ NDSS registrants, aged 18-75 years with non-insulin-treated T2D, who have previously consented to being contacted about research opportunities, will be invited to take part either via email $(n=10000)$ or postal mail $(n=2000)$ as per the registrants preferred method of contact. The NDSS is an Australian government initiative, administered by Diabetes Australia. The NDSS registry includes over 1.2 million Australians with T2D and is considered to be one of the most comprehensive and up-to-date diabetes prevalence data sets in Australians. ${ }^{36}$ The random sample will be stratified by state and territory to facilitate representation across Australia, ideally in line with population distribution across the eight states and territories. The research team will not have access to NDSS registrants' details unless they make contact/take part in the study, and the NDSS will not be notified of participating registrants. The total number of invited registrants was selected based on adoption of a conservative response rate of $8 \%,{ }^{37}$ and an expected $46 \%$ translation from consent to enrolled participant (as seen in the pilot $\mathrm{RCT}^{34}$ ). Invited NDSS registrants will receive an invitation reminder via e-mail or postal mail 2 weeks following first contact. If our target sample size is not reached within 4 weeks of the initial invitation, a second NDSS e-mail/mailout will be sent until our target sample size is reached or the 2-month recruitment period has concluded. The number of registrants contacted and method (e-mail vs mail) for subsequent recruitment efforts will be informed by the success rate from the original invitation (ie, percentage enrolled reporting hearing about the study via email or mail invitation). The study will also be advertised online via the researchers' affiliated professional websites and social media accounts, and a study flyer will be circulated to diabetes researcher and health professional networks.

\section{Study procedure}

The schedule of enrolment, intervention and assessment is detailed in Figure 1. Study recruitment will be open for a maximum of 2 months or until sample size (enrolled) is reached. Participation (from study entry to exit) will be for a duration of 6 months. Study advertisements will direct potential participants to the study website (hosted by Qualtrics) to access the Plain Language Statement, provide informed consent and complete screening questions online. Eligibility will be determined automatically based on responses. Eligible participants will be directed immediately to complete an online baseline survey, and, following submission, will be allocated at random to one of two study arms. Randomised participants will receive an email including details about how to access the relevant online resources for their study arm. For participants allocated to the intervention group, this will include a unique username and password enabling access. All participants will be asked to access their allocated resource(s) at their convenience within the following 2-week period, with no further instruction provided regarding the number of resource visits or length of time viewing the resources(s). One week following allocation, participants will receive a reminder email to access/log into the resource. Participants will be sent an email with a link to the online follow-up survey at 2 weeks and 6 months following baseline. The 2-week follow-up survey will be available for completion for 2 weeks, and the 6-month follow-up survey will be available for completion for 3 weeks. Study end point for all participants will be marked by either submission of the 6-month follow-up survey (within 21 days of request) or non-submission at 22 days following the survey request.

\section{Randomisation and blinding}

After baseline survey submission, participants will be stratified by gender (due to prior gender imbalance observed among participants recruited to related studies ${ }^{91}$ ) and randomised to either the intervention or control arm using computer-generated, randomly permuted block sizes of four, six or eight. The randomisation sequence will be computer generated and the allocation will be fully concealed from both the investigators and participants. On randomisation, participants will receive an email from a researcher, independent of the study investigator team and who does not have access to the incoming survey data (except for participant ID, name, gender and email address), specifying access details to their allocated online resource. The statistician, participants and investigator team will remained blinded to study arm allocation throughout data collection and analyses. The project manager (EEH), who will monitor incoming survey data, will be blinded from study arm allocation except where a participant self-identifies study arm allocation within the follow-up surveys (eg, in a free-text response box). Any breaches will be recorded and reported with the main findings.

\section{Intervention}

Intervention group participants will receive access to a novel psychoeducational web-based resource, 'Is insulin right for me?'. The intervention was developed using a systematic process grounded in behaviour change theory and has been described elsewhere. ${ }^{33}$ In brief, eight salient psychological barriers to insulin therapy were identified via literature search. Each barrier (ie, determinant of behaviour) was mapped to relevant domains of the theoretical domains framework. ${ }^{38}$ Determinants were then mapped onto BCTs considered relevant to overcoming the modifiable barriers. ${ }^{32}{ }^{38}$ Content responding to each barrier was developed by the investigator team (experts in health psychology, primary care medicine and diabetes education) and refined following consumer feedback (cognitive debriefing interviews, $\mathrm{n}=6$ ) and external 
expert peer review $(n=5)$ to ensure relevance for people with T2D and clinical accuracy.

The eight barriers targeted in the 'Is insulin right for $m e$ ' resource are phrased as common questions, with one barriers/question per website page (see table 1). The resource home page lists all eight barriers/questions as well as a preview (a key summary statement that responds to the question and content overview). The intervention is purposefully brief and self-directed, with the home page text asking which of eight questions about insulin are concerns for participants. For each selected barrier, an active intervention is presented on a separate webpage (200-500 words; $5 \mathrm{~min}$ read) to facilitate user engagement. In addition, the resource includes information about the key benefits of insulin therapy ${ }^{1}$ : that it lowers blood glucose levels ${ }^{2}$; can lower your risk of long-term health complications ${ }^{3}$; can make you feel better and ${ }^{4}$ can make managing your diabetes more flexible. The lesser focus on benefits than barriers is due to the evidence that most people with T2D experience/report barriers to insulin therapy despite endorsing benefits. ${ }^{14}{ }^{19}$ Finally, the resource also provides links to other resources about T2D and insulin available from the NDSS and study information.

\section{Control group}

Control arm participants will be directed to a static webpage including links to publicly available text-based NDSS factsheets, including: 'Insulin' and 'Medication for type 2 diabetes'. The control group webpage also includes links to further information about the study and research team (consistent with intervention arm).

\section{Outcomes}

The coprimary outcome measures are the difference in mean negative insulin appraisals, as measured by the Insulin Treatment Appraisal Scale (ITAS) Negative subscale score, ${ }^{39}$ between the intervention and control arm at 2-week and 6-month follow-up, adjusted by baseline scores. We hypothesise that, at 2 weeks, a statistically significant difference in mean ITAS negative scores of $\geq 4$ points (approximately 0.5 SD) will be observed between the intervention and control arm, favouring the intervention arm; and that this difference will be sustained at 6 months.

Our secondary outcome measures are immediate and sustained between-arm differences in: (a) positive insulin appraisals, as measured by ITAS positive subscale $\operatorname{score}^{39}$; and (b) hypothetical willingness to begin insulin therapy, as measured by a single item. ${ }^{35}$ We hypothesise that, at 2 weeks and 6 months, a statistically significant betweengroup difference will be observed in:

1. mean ITAS positive scores, adjusted for baseline scores, favouring the intervention arm.

2. The percentage of participants who respond 'not at all willing' (hypothetical willingness item). The intervention arm will be less likely to be 'not at all willing' compared with controls.
The following survey data will be examined by study arm for process evaluation purposes:

1. Clinical discussion and recommendation of insulin therapy, change in medications and satisfaction with diabetes management at 6-month follow-up

2. Change in secondary psychosocial outcome scores at 2-week and 6-month follow-up: diabetes-specific distress (PAID),${ }^{40}$ illness perceptions (BIPQ),${ }^{41}$ diabetesspecific self-efficacy (CIDS), ${ }^{42}$ study-specific insulinrelated knowledge questionnaire.

3. Diabetes-specific knowledge at baseline. ${ }^{43}$

4. Study-specific resource use and acceptability (study specific items) as 2-week follow-up.

Figure 1 details the self-reported demographic, clinical, psychosocial and study-specific data to be collected and the time points at which they are to be collected. In addition, website analytics data will be collected to assess protocol fulfilment with the intervention resource (ie, proportion of 'enrolled' participants who accessed the 'Is insulin right for me?' website at least once). Various analytics (eg, average number of online resource visits; time (minutes) spent on online resource; most commonly (frequency, $\%$ ) viewed pages) will be examined to explore any relationship(s) between type/duration of content accessed and the study outcomes. Finally, number of views and average time spent watching two videos embedded in the intervention resource will be captured via YouTube.

\section{Sample size}

Using a power analysis for repeated measures analysis of variance, a minimum sample size of $\mathrm{N}=250 \quad(\mathrm{n}=125$ per $\mathrm{arm})$ is required to detect a minimally important difference of half a standard deviation $(\mathrm{SD}=9)$ in ITAS Negative Scores ${ }^{39}$ between study arms with a correlation of 0.65 between repeated measures, at $85 \%$ power and 0.05 significance level using a two-sided test. Assuming a 20\% attrition rate at 2 weeks $^{34}$ and a further $20 \%$ attrition at 6 months, the targeted sample size inflates to approximately $\mathrm{N}=392$ ( $\mathrm{n}=196$ per arm). Overall, a $40 \%$ attrition rate is incorporated into our estimated sample size and replacements will not be made for losses to follow-up.

\section{Data collection, management and analysis}

Participant-reported data will be collected online via Qualtrics, hosted through the Deakin University secure network. Consent, eligibility screening and baseline survey data will be collected in a single sitting (directed via study advertisement link) and an email will provide enrolled participants with a link to online follow-up surveys. The intervention website will require participant log-in, allowing for automatic collection of website usage data for each intervention participant via Google Analytics.

To improve participant retention and protocol compliance, trial participants will receive reminder emails to access/view the allocated online resource (sent to all participants 2 weeks following allocation. In addition, reminder emails will be sent at 1 week (and 2 weeks 
Table 1 Description of the eight barriers targeted in the 's insulin right for me?' resource

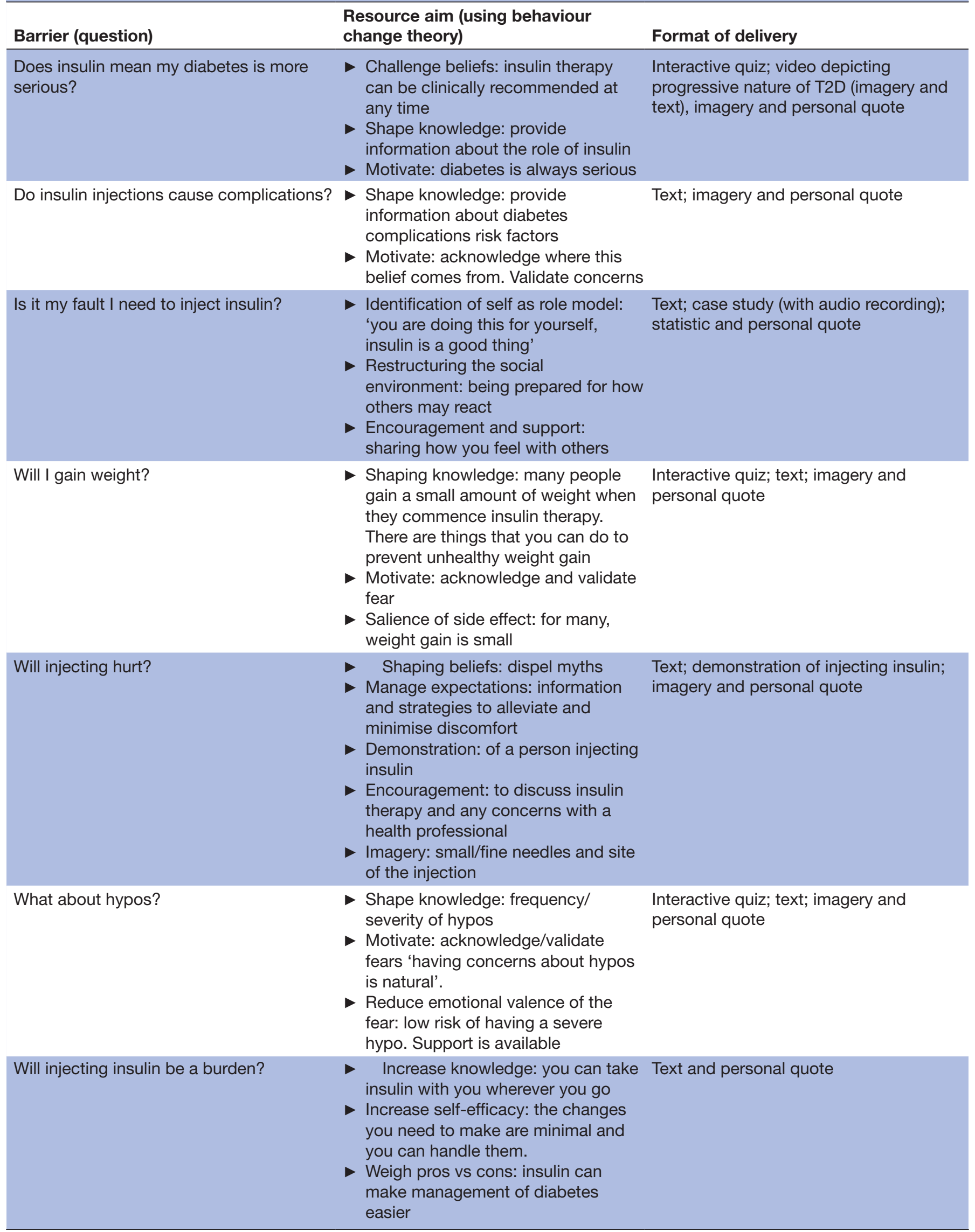


Table 1 Continued

Barrier (question)

What will others think of me?

\section{Resource aim (using behaviour} change theory)

- Identification of self as role model: 'you are doing this for yourself, insulin is a good thing'

- Restructuring the social environment: being prepared for how others may react

- Encouragement and support: start a 'safe' conversation to share how you feel with others

\section{Format of delivery}

Case studies with examples (with audio recording); text; and personal quote for 6-month time- oint) to participants who have yet to commence their online follow-up surveys. To aid recruitment and retention, participants who complete all three surveys (the baseline, 2-week and 6-month follow-ups) will be entered into a prize draw to win one of 20 AUD $\$ 100$ e-gift vouchers.

Participants who do not access their allocated resource(s) will still be followed up until the end of the trial unless they withdraw from the trial. Participants who do not complete the 2-week follow-up survey will have 'missing data' at 2 weeks, but remain eligible to complete the 6-month follow-up survey. Participants who do not complete the 6-month follow-up survey within 3 weeks of receipt will have 'missing data' at 6 months. Participants with missing data at both follow-up time points will be deemed 'lost to follow-up'.

Study data collected from withdrawn participants will be deleted, with the exception of basic deidentified sample characteristics (gender, age, diabetes duration), trial arm allocation, timing of withdrawal and reason for withdrawal, where applicable.

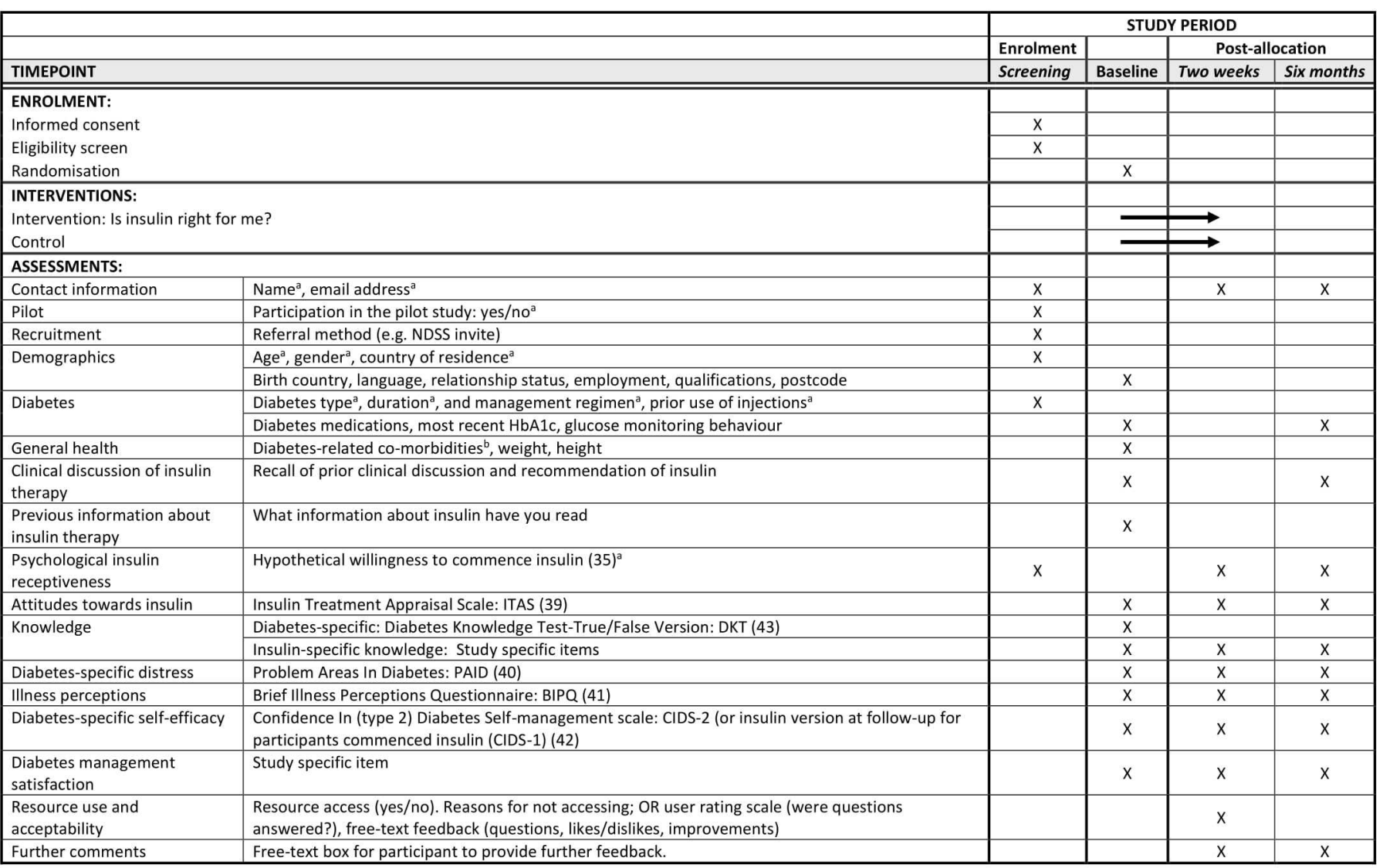

Figure 1. Schedule of enrolment, interventions, and assessments. a-Compulsory questions for participation. b-Comorbidities included: kidney disease, retinopathy, neuropathy, heart disease, stroke, vascular disease, sexual dysfunction, other (to be specified). HbA1c, haemoglobin A1C; NDSS; National Diabetes Services Scheme. 


\section{Data storage}

At study conclusion, survey data and website usage data (for intervention participants only) will be downloaded from Qualtrics and Google Analytics, respectively, and linked according to participant ID. Identifiable information (email, name) will be separated from study data and stored along with participant ID number in a passwordencrypted excel spreadsheet. All data will be stored in a secure electronic file accessible only by the research team. In accordance with clinical trial regulations, data will be kept for a minimum of 15 years after study completion and then disposed by erasing of electronic files.

\section{Statistical methods}

Quantitative data analyses will be performed using Stata/ SE V.16.0 and/or IBM SPSS V.26. Descriptive statistics will be used to describe participant baseline characteristics and psychological outcomes at each time point. Participant characteristics at baseline will be visually assessed by allocation for imbalance. The overall characteristics of the study cohort will be compared with those lost to follow-up.

An intention-to-treat approach will be adopted, whereby participants will be analysed according to the arm they were allocated to, and all participants will be included in the analysis. A linear mixed effects model will be used to estimate the difference in mean ITAS negative scores between arms at 2 weeks and 6 months using restricted maximum likelihood estimation. Treatment arm, all three time points (baseline, 2 weeks and 6 months), and the interaction between treatment arm and time points will be included as fixed effects in the model. Random effects will be used to account for repeated participant measures. The outcome measure will be adjusted by age, diabetes duration and education should these be imbalanced between the arms at baseline. As a sensitivity analysis, pattern mixture models will be used to determine whether study conclusions from the analyses described above would change should data be missing not at random.

ITAS positive scores (secondary outcome) and continuous psychosocial process evaluation outcomes (eg, PAID, BIPQ, CIDS) will be analysed using the same modelling approached described above. An ordinal logistic mixed effects model will be used to quantify between-arm differences in the willingness to begin insulin therapy (secondary outcome) at various time points.

Descriptive data will be used to explore trends in protocol fulfilment, website analytics and acceptability data as well as medication changes and clinical discussion of insulin therapy at 6 months separately for each study arm.

\section{Monitoring}

Coauthors EH-T and JS are the responsible investigators and will oversee the research project. During recruitment and data collection, the number of potential participants consenting, eligible and enrolled as well as dates of all participant encounters (ie, enrolment; intervention access \& reminder emails; survey access, reminder and closure) and survey completion will be monitored by EEH and communicated to investigator team. The primary funding body will be allowed access to all deidentified data from the study for audit purposes, if requested.

This research protocol does not include administration or manipulation of, or investigation of the effects of, any pharmacological or therapeutic goods. However, in line with the pharmacovigilance reporting requirements of the funding body, all survey data collected will be screened for adverse events that may be associated with the funding body's products and, in the event of the research team becoming aware of a potential adverse event, participants will be contacted (via email) and invited to respond to additional questions about this event (eg, medication brand name, dose and timing, healthcare utilisation symptoms, other consequences). Nonresponse will not affect participation in the study proper. Deidentified information obtained about the event will be submitted to the funder and, if relevant, the Australian Therapeutic Goods Administration.

\section{Patient and public involvement}

People with T2D were involved in the review and iterative refinement of the intervention content and design. This involved cognitive debriefing interviews with six adults with T2D to review draft content during intervention development, for which the findings and consequential refinements are detailed elsewhere. ${ }^{33}$ In addition, user ratings and qualitative feedback were provided by 13 pilot RCT participants who were allocated to the intervention. ${ }^{34}$ Refinements made to the intervention following piloting included, for example, improving website navigation between barrier webpages and the addition of 'print-friendly' downloadable Portable Document Format (PDF) content. ${ }^{34}$ People with T2D were not involved in the development of the study design, nor will they be involved in conduct of the study or dissemination of the study findings.

\section{Ethics and dissemination}

This trial received ethical approval Deakin University Human Research Ethics Committee (Ref: 2020-073). This study will be conducted in compliance with this protocol (V.SA2017-11697; V2.2e 16 June 2020), which is registered with the Australian New Zealand Clinical Trials Registry (ACTRN: 12621000191897, registered 23 February 2021). Note, this protocol was submitted for registration on 10 December 2020, prior to recruitment commencement (11 January 2021), though approved retrospectively following enrolment of the first participant and prior to last participant enrolment. Any changes to the protocol will be communicated to the human research ethics committee, funder and trial register. Protocol registration will be updated with any approved amendments to the protocol, and protocol departures will be documented in any reports or manuscripts resulting from this study.

Potential participants view the study plain language form online (online supplemental file 1) and must indicate consent (by ticking a box) prior to participating. Participants 
are free to withdraw from the study at any time, and for any reason, prior to completion of data collection.

The findings will be prepared for academic presentation at scientific meetings and in peer-reviewed journals. A lay summary of findings will be published on the research team's website and disseminated via e-newsletter. Study findings will also be reported to the funding body.

Deidentified data may be made available, on request, to the funding body.

\section{DISCUSSION}

This RCT will provide high-quality evidence regarding the efficacy and acceptability of a novel, web-based resource: 'Is insulin right for me?'. Using best-practice intervention development principles and evaluation guidance, ${ }^{33} 34$ the intervention was designed to reduce salient psychological barriers to insulin, which are extremely common among people with T2D and associated with deleterious delay of insulin uptake. ${ }^{144}$ To our knowledge, this study will be the first fully powered RCT conducted to test the impact of any intervention specifically designed to address salient psychological barriers to insulin among adults with T2D, reporting some level of psychological insulin resistance.

The described study will provide evidence of the acceptability of this web-based resource among Australians with T2D, who report some level of psychological insulin resistance, which may inform real-world implementation strategies and further refinements as required. A potential limitation of this trial is the expected low response rate and self-selection bias of the sample recruited via an invitation from the NDSS, which may not be representative of those most in need (ie, those with a high HbAlc yet not at all willing to commence insulin) as well as linguistically diverse communities. Participants' demographic characteristics (eg, gender, state/territory, language, country of birth) will be compared with the general Australian population of adults with T2D to examine the representativeness of the sample. If the intervention is shown to be efficacious, further research will be warranted to investigate its impact on timely insulin uptake (and consequently on HbAlc) as well as the feasibility of implementation in primary care settings among adults with T2D for whom treatment intensification is clinically indicated.

\section{Author affiliations}

${ }^{1}$ School of Psychology, Deakin University, Geelong, Victoria, Australia

${ }^{2}$ The Australian Centre for Behavioural Research in Diabetes, Diabetes Victoria,

Melbourne, Victoria, Australia

${ }^{3}$ Department of General Practice, University of Melbourne, Carlton, Victoria, Australia

${ }^{4}$ School of Nursing and Midwifery, Deakin University, Burwood, Victoria, Australia

${ }^{5}$ Rural Health School, La Trobe University, Bendigo, Victoria, Australia

${ }^{6}$ Department of Psychology, University of Copenhagen, Kobenhavn, Denmark

Twitter Elizabeth Holmes-Truscott @holmestruscott and Jane Speight @ janespeight

Acknowledgements We thank the participants with T2D who participated in intervention development and piloting phases. We thank Victoria Yutronich (The Australian Centre for Behavioural Research in Diabetes, Diabetes Victoria) for website design and technical support and Shaira Baptista (Deakin University for research assistance.
Contributors EH-T and JS conceived of the intervention and the described program of research. EH-T and JS developed the study protocol, with input from EEH, HMH, JF, VH and TS. EEH, JS, TS and EH-T led the development of the intervention, with contributions from JF, and VH. HMH calculated the sample size and developed the statistical analysis plan. EH-T was responsible for drafting the manuscript, which EEH, HMH, JF, VH, TS and JS reviewed and contributed to. All authors approved the final manuscript.

Funding This work was supported by an instigator-sponsored-study grant from Sanofi-Aventis Australia Pty Ltd (Sanofi). Sanofi was not involved in the study design and will not be involved in the collection, analysis or interpretation of the study data, but was given the opportunity to view the manuscript prior to submission. The decision to submit for publication was made independently by the authors. Sanofi will be allowed access to all de-identified data from the study for research and audit purposes, if requested. Costs associated with participation incentives, website development and data management were funded (in full, or partially) by the Australian Centre for Behavioural Research in Diabetes (ACBRD). In-kind support including project oversight was provided by the Investigator team. JS is supported by the core funding to the ACBRD provided by the collaboration between Diabetes Victoria and Deakin University. EHT was supported by the same plus a Deakin University Deans Research Postdoctoral Fellowship (2018-2020).

Competing interests EH-T has undertaken research funded by an unrestricted educational grant from Abbott Diabetes Care, AstraZeneca, and Sanofi; received speaker fees from Novo Nordisk and Roche to Australian Centre for Behavioural Research in Diabetes (ACBRD); and served on an advisory board for AstraZeneca. EEH has no conflicts of interest to disclose. JF has received unrestricted educational grants for research support from Roche, Sanofi, and Medtronic. TS serves on advisory boards for Novo Nordisk and Liva Health Care and is currently on a EIT Health research grant held jointly with Roche Diagnostics. JS has served on advisory boards for Janssen, Medtronic, Roche Diabetes Care, and Sanofi Diabetes; her research group (Australian Centre for Behavioural Research in Diabetes [(ACBRD])) has received honoraria for this advisory board participation and has also received unrestricted educational grants and in-kind support from Abbott Diabetes Care, AstraZeneca, Medtronic, Roche Diabetes Care, and Sanofi Diabetes. JS has also received sponsorship to attend educational meetings from Medtronic, Roche Diabetes Care, and Sanofi Diabetes, and consultancy income or speaker fees from Abbott Diabetes Care, AstraZeneca, Medtronic, Novo Nordisk, Roche Diabetes Care, and Sanofi Diabetes. All other authors have no conflicts of interest to declare.

Patient consent for publication Not applicable.

Provenance and peer review Not commissioned; externally peer reviewed.

Supplemental material This content has been supplied by the author(s). It has not been vetted by BMJ Publishing Group Limited (BMJ) and may not have been peer-reviewed. Any opinions or recommendations discussed are solely those of the author(s) and are not endorsed by BMJ. BMJ disclaims all liability and responsibility arising from any reliance placed on the content. Where the content includes any translated material, BMJ does not warrant the accuracy and reliability of the translations (including but not limited to local regulations, clinical guidelines, terminology, drug names and drug dosages), and is not responsible for any error and/or omissions arising from translation and adaptation or otherwise.

Open access This is an open access article distributed in accordance with the Creative Commons Attribution Non Commercial (CC BY-NC 4.0) license, which permits others to distribute, remix, adapt, build upon this work non-commercially, and license their derivative works on different terms, provided the original work is properly cited, appropriate credit is given, any changes made indicated, and the use is non-commercial. See: http://creativecommons.org/licenses/by-nc/4.0/.

\section{ORCID iDs}

Elizabeth Holmes-Truscott http://orcid.org/0000-0001-9139-4663

Edith E Holloway http://orcid.org/0000-0002-1343-9982

Hanafi M Husin http://orcid.org/0000-0002-3793-3221

John Furler http://orcid.org/0000-0003-0339-5848

Virginia Hagger http://orcid.org/0000-0003-3845-2814

Timothy C Skinner http://orcid.org/0000-0002-0018-6963

Jane Speight http://orcid.org/0000-0002-1204-6896

\section{REFERENCES}

1 Davies MJ, D'Alessio DA, Fradkin J, et al. Management of hyperglycemia in type 2 diabetes, 2018. A consensus report by the American diabetes association (ADA) and the European association for the study of diabetes (EASD). Diabetes Care 2018;41:2669-701. 
2 Gunton JE, Cheung NW, Davis TME, et al. A new blood glucose management algorithm for type 2 diabetes: a position statement of the Australian diabetes Society. Med J Aust 2014;201:650-3.

3 Inzucchi SE, Bergenstal RM, Buse JB, et al. Management of hyperglycemia in type 2 diabetes, 2015: a patient-centered approach: update to a position statement of the American diabetes association and the European association for the study of diabetes. Diabetes Care 2015;38:140-9.

4 Paul SK, Klein K, Thorsted BL, et al. Delay in treatment intensification increases the risks of cardiovascular events in patients with type 2 diabetes. Cardiovasc Diabetol 2015;14:100.

5 Holman RR, Paul SK, Bethel MA, et al. 10-Year follow-up of intensive glucose control in type 2 diabetes. N Engl J Med Overseas Ed 2008;359:1577-89.

6 Khunti S, Davies MJ, Khunti K. Clinical inertia in the management of type 2 diabetes mellitus: a focused literature review. British Journal of Diabetes 2015;15:65-9.

7 Khunti S, Khunti K, Seidu S. Therapeutic inertia in type 2 diabetes: prevalence, causes, consequences and methods to overcome inertia. Ther Adv Endocrinol Metab 2019;10:2042018819844694.

8 Khunti K, Wolden ML, Thorsted BL, et al. Clinical inertia in people with type 2 diabetes: a retrospective cohort study of more than 80,000 people. Diabetes Care 2013;36:3411-7.

9 Furler J, O'Neal D, Speight J, et al. Supporting insulin initiation in type 2 diabetes in primary care: results of the stepping up pragmatic cluster randomised controlled clinical trial. BMJ 2017;356:j783.

10 Manski-Nankervis J-A, Furler J, O'Neal D, O'Neal D, et al. Overcoming clinical inertia in insulin initiation in primary care for patients with type 2 diabetes: 24-month follow-up of the stepping up cluster randomised controlled trial. Prim Care Diabetes 2017;11:474-81.

11 Zafar A, Stone MA, Davies MJ, et al. Acknowledging and allocating responsibility for clinical inertia in the management of type 2 diabetes in primary care: a qualitative study. Diabet Med 2015;32:407-13.

12 Furler J, Spitzer O, Young D, et al. Insulin in general practice barriers and enablers for timely initiation. Aust Fam Physician 2011;40:617-21.

13 Gabbay RA, Kendall D, Beebe C, et al. Addressing therapeutic inertia in 2020 and beyond: a 3-year initiative of the American diabetes association. Clin Diabetes 2020;38:371-81.

14 Holmes-Truscott E, Furler J, Blackberry I, et al. Predictors of insulin uptake among adults with type 2 diabetes in the stepping up study. Diabetes Res Clin Pract 2017;133:204-10.

15 Wrzal PK, Bunko A, Myageri V, et al. Strategies to overcome therapeutic inertia in type 2 diabetes mellitus: a scoping review. Can $J$ Diabetes 2021;45:273-281.e13.

16 Polonsky WH, Fisher L, Hessler D, et al. Identifying solutions to psychological insulin resistance: an international study. J Diabetes Complications 2019;33:307-14.

17 Or KY, Yip BH-K, Lau CH, et al. Peer education group intervention to reduce psychological insulin resistance: a pilot Mixed-Method study in a Chinese population. Diabetes Ther 2018;9:113-24.

18 Kuo CR, Quan J, Kim S, et al. Group visits to encourage insulin initiation: targeting patient barriers. J Clin Nurs 2017;26:1705-13.

19 Holmes-Truscott E, Blackberry I, O'Neal DN, et al. Willingness to initiate insulin among adults with type 2 diabetes in Australian primary care: results from the stepping up study. Diabetes Res Clin Pract 2016;114:126-35.

20 Upsher R, Allen-Taylor M, Reece I, et al. Experiences of attending group education to support insulin initiation in type 2 diabetes: a qualitative study. Diabetes Ther 2020;11:119-32.

21 Holmes-Truscott E, Browne JL, Speight J. The impact of insulin therapy and attitudes towards insulin intensification among adults with type 2 diabetes: a qualitative study. J Diabetes Complications 2016;30:1151-7.

22 Holmes-Truscott E, Skinner TC, Pouwer F, et al. Negative appraisals of insulin therapy are common among adults with Type 2 diabetes using insulin: Results from Diabetes MILES - Australia crosssectional survey. Diabet Med 2015;32:1297-303. n/a-n/a.

23 Davies MJ, Gagliardino JJ, Gray LJ, et al. Real-World factors affecting adherence to insulin therapy in patients with type 1 or type 2 diabetes mellitus: a systematic review. Diabet Med 2013;30:512-24.
24 Holmes-Truscott E, Pouwer F, Speight J. Further investigation of the psychometric properties of the insulin treatment appraisal scale among insulin-using and non-insulin-using adults with type 2 diabetes: results from diabetes MILES-Australia. Health Qual Life Outcomes 2014;12:87.

25 Brod M, Alolga SL, Meneghini L. Barriers to initiating insulin in type 2 diabetes patients: development of a new patient education tool to address myths, misconceptions and clinical realities. Patient 2014;7:437-50.

26 Patel N, Stone MA, Hadjiconstantinou M, et al. Using an interactive DVD about type 2 diabetes and insulin therapy in a UK South Asian community and in patient education and healthcare provider training. Patient Educ Couns 2015;98:1123-30.

27 Horigan G, Davies M, Findlay-White F, et al. Reasons why patients referred to diabetes education programmes choose not to attend: a systematic review. Diabet Med 2017;34:14-26.

28 Brewster S, Bartholomew J, Holt RIG, et al. Non-attendance at diabetes outpatient appointments: a systematic review. Diabetic Medicine 2020;37:1427-42.

29 Lui C-W, Col JR, Donald M, et al. Health and social correlates of Internet use for diabetes information: findings from Australia's living with diabetes study. Aust J Prim Health 2015;21:327-33.

30 van Vugt M, de Wit M, Cleijne WHJJ, et al. Use of behavioral change techniques in web-based self-management programs for type 2 diabetes patients: systematic review. J Med Internet Res 2013;15:e279.

31 Abraham C, Michie S. A taxonomy of behavior change techniques used in interventions. Health Psychol 2008;27:379-87.

32 Michie S, Richardson M, Johnston M, et al. The behavior change technique taxonomy (V1) of 93 hierarchically clustered techniques: building an international consensus for the reporting of behavior change interventions. Ann Behav Med 2013;46:81-95.

33 Holloway EE, Speight J, Furler J, et al. 'Is Insulin Right for Me?' Development of a theory-informed, web-based resource for reducing psychological barriers to insulin therapy in type 2 diabetes. BMJ Open 2021;11:e045853.

34 Holmes-Truscott E, Holloway EE, Husin HM, et al. 'Is insulin right for me?': Feasibility of a pilot randomised controlled trial and acceptability of a web-based intervention to reduce psychological barriers to insulin therapy among adults with type 2 diabetes. Diabet Med 2021;41.

35 Polonsky WH, Hajos TRS, Dain M-P, et al. Are patients with type 2 diabetes reluctant to start insulin therapy? an examination of the scope and underpinnings of psychological insulin resistance in a large, International population. Curr Med Res Opin 2011;27:1169-74.

36 Australian Institute of Health and Welfare. Diabetes prevalence in Australia: an assessment of national data sources. Canberra: AlHW, 2009.

37 Speight J, Browne JL, Holmes-Truscott E, et al. Diabetes MILESAustralia (management and impact for long-term empowerment and success): methods and sample characteristics of a national survey of the psychological aspects of living with type 1 or type 2 diabetes in Australian adults. BMC Public Health 2012;12.

38 Cane J, O'Connor D, Michie S. Validation of the theoretical domains framework for use in behaviour change and implementation research. Implement Sci 2012;7:37.

39 Snoek FJ, Skovlund SE, Pouwer F. Development and validation of the insulin treatment appraisal scale (ITAs) in patients with type 2 diabetes. Health Qual Life Outcomes 2007;5.

40 Polonsky WH, Anderson BJ, Lohrer PA, et al. Assessment of diabetes-related distress. Diabetes Care 1995;18:754-60.

41 Broadbent E, Petrie KJ, Main J, et al. The brief illness perception questionnaire. J Psychosom Res 2006;60:631-7.

42 PolonskyWH, FisherL, SnoekF. Evaluation of the confidence in diabetes scale (CIDS-2) for patients with poorly controlled T2DM. American Diabetes Association's 69th Scientific Sessions. New Orleans, 2009.

43 Fitzgerald JT, Funnell MM, Anderson RM, et al. Validation of the revised brief diabetes knowledge test (DKT2). Diabetes Educ 2016;42:178-87.

44 Odawara M, Ishii $\mathrm{H}$, Tajima N, et al. Impact of patient attitudes and beliefs to insulin therapy upon initiation, and their attitudinal changes after initiation: the dawn Japan study. Curr Med Res Opin 2016;32:681-6. 